\title{
L'entrepreneuriat féminin en milieu rural : Le cas du Fonds AFER Canada
}

\author{
Pierre Simard et Julie Fortin \\ École nationale d'administration publique \\ Université du Québec
}

\section{Introduction}

Même si la courbe de croissance du nombre de femmes entrepreneures est à la hausse, elles sont toujours minoritaires dans le monde des affaires : elles représentent environ le tiers des entrepreneurs au Québec ${ }^{1}$. Pour pallier ce déséquilibre, plusieurs initiatives visant à stimuler l'entrepreneuriat féminin ont vu le jour. Le Fonds AFER Canada en fait partie.

Mis de l'avant par le Réseau des Sociétés d'aide au développement des collectivités du Québec (SADC) en collaboration avec Développement économique Canada, ce projet pilote - maintenant terminé ciblait l'entrepreneuriat féminin en milieu rural. Son intervention a pris deux formes distinctes, soit l'octroi d'une aide financière non remboursable pour le développement et le démarrage de l'entreprise ainsi qu'une aide technique appropriée aux besoins des bénéficiaires (soutien, conseils, accompagnement).

Afin de documenter les retombées de cette initiative et de juger de la pertinence de l'étendre à l'ensemble des territoires desservis par ses membres, le Réseau des SADC du Québec nous a confié l'évaluation du projet pilote ${ }^{2}$. Dans le cadre de cette évaluation, 130 bénéficiaires ont été interrogées lors d'une enquête téléphonique, soit plus de $80 \%$ de 1 'ensemble des bénéficiaires ${ }^{3}$. Des études de cas ont aussi été réalisées auprès de deux SADC ayant participé au projet ${ }^{4}$. Cet article présente les principales observations issues de cette évaluation et les leçons à tirer de la mise en œuvre de ce projet pilote.

\section{Le Fonds AFER Canada, une intervention en deux phases}

Le Fonds AFER Canada a été déployé autour des deux grandes phases caractéristiques de la création d'une entreprise: le développement du projet d'entreprise (phase 1) et le démarrage de l'entreprise proprement dit (phase 2).

\section{Phase 1 : Développement du projet et réalisation du plan d'affaires}

La phase $1 \mathrm{du}$ Fonds AFER Canada prévoyait l'octroi d'une aide financière maximale de $3000 \$$ pour aider les bénéficiaires à développer leur projet d'entreprise et à réaliser un plan d'affaires. De plus, des services d'accompagnement et de soutien technique étaient offerts par les SADC: recherche d'information pertinente sur le secteur d'activité visé par le projet d'entreprise, élaboration de prévisions budgétaires, mise en forme du plan d'affaires, etc. Ces services étaient variables d'une région à l'autre en fonction des besoins des bénéficiaires et, bien sûr, des champs d'expertise des intervenants SADC $^{5}$.

\section{Phase 2 : Démarrage de l'entreprise}

La phase 2 du Fonds AFER Canada visait à soutenir les bénéficiaires dans les activités liées au démarrage de leur entreprise, comme la recherche de financement, l'achat d'équipement, la mise en mar- 
ché d'un produit, la publicité, etc. Les bénéficiaires pouvaient recevoir une aide financière maximale de $25000 \$$ pour cette phase du projet. Une aide technique (soutien, conseils) était également offerte pour accompagner les bénéficiaires dans leurs démarches.

Il est important de noter que les bénéficiaires de la phase 1 n'étaient pas automatiquement admises à la phase 2 puisque le projet développé devait rencontrer les conditions d'admissibilité du Fonds AFER Canada. Pour être admissible, la bénéficiaire ne devait pas être déjà propriétaire d'une entreprise. De plus, le projet d'entreprise devait s'inscrire dans un « secteur de niche ${ }^{6}$. Il était également possible que des bénéficiaires soient admises directement à la phase 2 du projet pilote si elles avaient déjà en main un plan d'affaires.
Enfin, outre les deux grandes phases citées plus haut, le Fonds AFER Canada s'est accompagné d'activités de promotion de l'entrepreneuriat en milieu rural. Des sessions publiques de sensibilisation à l'entrepreneuriat ont aussi été offertes aux femmes intéressées.

\section{Le profil des bénéficiaires}

L'enquête révèle que les bénéficiaires du Fonds AFER Canada sont surtout des femmes âgées de 25 à 54 ans (tableau 1). Leur scolarisation est majoritairement de niveau postsecondaire (tableau 3) puisque les détentrices d'une formation universitaire $(31,6 \%)$ et collégiale $(31,5 \%)$ représentent près des deux tiers des répondants.

\section{Tableau 1 : Répartition des bénéficiaires du Fonds AFER Canada en fonction de l'âge}

\begin{tabular}{lcc} 
Catégorie d'âge & Fréquence & $\mathbf{\%}$ \\
\hline 18 à 24 ans & 4 & 3,1 \\
25 à 34 ans & 41 & 31,5 \\
35 à 44 ans & 46 & 35,4 \\
45 à 54 ans & 33 & 25,4 \\
55 à 64 ans & 5 & 3,8 \\
65 ans et plus & 1 & 0,8 \\
Total & 130 & 100,0 \\
\hline
\end{tabular}

Tableau 2 : Répartition des bénéficiaires du Fonds AFER Canada selon le niveau de scolarité

\begin{tabular}{lcc} 
Dernier niveau de scolarité complété & Fréquence & \% \\
\hline Secondaire non complété & 15 & 11,5 \\
Diplôme d'études secondaires & 20 & 15,4 \\
Diplôme d'études professionnelles & 13 & 10,0 \\
Diplôme d'études collégiales général & 15 & 11,5 \\
Diplôme d'études collégiales techniques & 26 & 20,0 \\
Baccalauréat & 27 & 20,8 \\
Maîtrise & 13 & 10,0 \\
Doctorat & 1 & 0,8 \\
Total & 130 & 100,0
\end{tabular}




\section{L'aide et les services reçus}

Plus de $80 \%$ des répondantes de l'enquête (108) ont bénéficié d'une aide pour le démarrage de leur entreprise (phase 2). De ce nombre, 62 femmes $(47,7 \%)$ ont cumulé l'aide disponible en vertu des phases 1 et 2 du Fonds AFER Canada. Plus du tiers des répondantes, soit $35,4 \%$, n'ont bénéficié que de la phase $2 \mathrm{du}$ Fonds AFER Canada, soit l'aide au démarrage de l'entreprise.

\section{Tableau 3 : Répartition des répondantes selon les services reçus du Fonds AFER Canada}

\begin{tabular}{lcc} 
Services reçus & Fréquence & \% \\
\hline Phase 1 : aide pour le développement du projet & 22 & 16,9 \\
Phases 1 et 2 : aide pour le développement du projet & & \\
et le démarrage de l'entreprise & 62 & 47,7 \\
Phase 2: aide pour le démarrage de l'entreprise & 46 & 35,4 \\
Total & 130 & 100,0
\end{tabular}

Bien que plusieurs bénéficiaires ne se soient pas prévalues de la phase $1 \mathrm{du}$ Fonds, la majorité d'entre elles ont toutefois pu bénéficier d'aides extérieures pour le développement de leur projet. En fait, plusieurs bénéficiaires interrogées ont déclaré avoir obtenu une aide complémentaire provenant d'autres programmes ou organismes, et ce, tant pour la réalisation du plan d'affaires que pour le démarrage de l'entreprise. Cette aide complémentaire, technique ou financière, provenait de sources variées: autres programmes des SADC (fonds d'investissement, stratégie jeunesse), centre local de développement, commission scolaire, carrefour jeunesse-emploi, Financière agricole, Secrétariat aux affaires autochtones, Emploi-Québec, Ministère de l'Agriculture, des Pêcheries et de l'Alimentation, Tourisme Québec, etc.

Enfin, un nombre moins important de répondantes, soit 22 (16,9\%), n'ont bénéficié que de l'aide prévue en vertu de la phase $1 \mathrm{du}$ Fonds AFER Canada. Comme mentionné précédemment, elles ont pu s'être vu refuser l'admissibilité à la phase 2. Toutefois, plusieurs bénéficiaires ont choisi d'abandonner leur projet pour différentes raisons (obtention d'un emploi, considérations familiales, etc.).

L'évaluation nous a aussi permis d'estimer le nombre d'heures d'accompagnement et d'aide technique dispensée par les SADC aux bénéficiaires du Fonds. Bien que la quantité d'aide technique rapportée soit évidemment plus grande chez les femmes qui ont reçu des services dans le cadre des deux phases du projet, près des deux tiers des répondants estiment cette aide à moins de 20 heures.

\section{Les obstacles liés à la création d'une entre- prise}

Afin de mieux connaître la situation des entrepreneures ciblées par le Fonds AFER Canada, l'évaluation a tenté d'identifier les principaux obstacles ou difficultés rencontrés par les bénéficiaires pendant leurs démarches.

Bien que les difficultés évoquées soient diverses, ce sont les problèmes liés à la recherche de financement qui reviennent le plus souvent: difficulté à obtenir les fonds nécessaires au démarrage de l'entreprise, difficulté à emprunter, difficulté à payer les factures, etc. Viennent ensuite les obstacles liés au manque de connaissances sur différents aspects de l'entrepreneuriat (plan d'affaires, démarrage, lois et règlements, comptabilité, etc.), au développement de marché et à la recherche de clients, à la gestion de l'entreprise, à l'application des lois, règlements ou normes (problèmes de zonage notamment) et, enfin, à la disponibilité des installations et équipements.

\section{Les retombées du Fonds AFER Canada sur l'entrepreneuriat féminin}

Les résultats du projet pilote ont été largement documentés lors de l'évaluation. Ces résultats montrent que le Fonds AFER Canada a contribué au 
développement du potentiel entrepreneurial de plusieurs femmes en région, à l'accès au financement ainsi qu'à la création d'entreprises et d'emplois.

$\mathrm{Au}$ moment de l'enquête, 101 entreprises avaient été démarrées par des bénéficiaires du Fonds AFER
Canada, créant 273 emplois (88 emplois à temps complet et 66 emplois saisonniers ou à temps partiel). Dans la première année d'opération, ces entreprises ont généré ou généreront un chiffre d'affaires variant de 0 à $150000 \$$.

\section{Tableau 5 : Création d'entreprises et d'emplois par les bénéficiaires du Fonds AFER Canada}

\begin{tabular}{lc} 
Indicateur & Fréquence \\
\hline Nombre d'entreprises créées & 101 \\
Nombre d'emplois créés & 273 \\
- emplois à temps complet & 88 \\
- emplois saisonniers ou à temps partiel & 66 \\
Nombre d'entreprises potentielles supplémentaires $^{7}$ & 7
\end{tabular}

Tableau 6 : Estimation du chiffre d'affaires pour la $1^{\text {re }}$ année d'opération

\begin{tabular}{lcc} 
Chiffre d'affaires & Fréquence & \% \\
\hline Moins de $10000 \$$ & 23 & 22,5 \\
$10000 \$$ à $19999 \$$ & 20 & 19,6 \\
$20000 \$$ à $29999 \$$ & 11 & 10,8 \\
$30000 \$$ à $39999 \$$ & 11 & 10,8 \\
$40000 \$$ à $49999 \$$ & 9 & 8,8 \\
50000 \$ et plus & 28 & 27,4 \\
Total $^{8}$ & 102 & 100,0
\end{tabular}

Ces données doivent être interprétées avec prudence. On ne peut attribuer entièrement la création de ces entreprises et de ces emplois à la seule intervention du Fonds AFER Canada. Dans certains cas, on l'a vu, les femmes entrepreneures n'ont bénéficié que de la phase 1 du Fonds, dans d'autres, elles ont obtenu de l'aide technique ou financière extérieure. Cela dit, la majorité des répondantes ont tout de même révélé que le Fonds AFER Canada a été déterminant dans la création de leur entreprise. En effet, au-delà de la création d'emplois et d'entreprises, tant les bénéficiaires que les intervenantes des SADC rencontrées estiment que le Fonds AFER Canada est responsable de plusieurs retombées.

De l'avis de la plupart des bénéficiaires, l'aide financière reçue a été déterminante dans la réalisation de leur projet. Une grande proportion d'entre elles considèrent qu'elles n'auraient pu mener à bien leur projet d'entreprise sans cette aide. Plus de $97 \%$ des bénéficiaires des phases 1 et 2 estiment que l'aide financière obtenue a non seulement été utile à la réalisation de leur projet, mais leur a permis d'accéder à un financement qu'il aurait été difficile d'obtenir autrement. Ainsi, pour certaines bénéficiaires, les sommes économisées en ce qui a trait au remboursement du capital et des intérêts auront permis d'investir dans la publicité et la mise en marché de leurs produits. Pour d'autres, l'aide financière aura permis de dégager la mise de fond nécessaire à l'obtention d'un financement provenant d'autres sources.

En outre, près de $85 \%$ des répondantes disent avoir acquis, grâce au Fonds AFER Canada, des connaissances et des habiletés indispensables à la création d'une entreprise. Elles considèrent avoir amélioré leurs compétences entrepreneuriales. Pour elles, les compétences acquises grâce à l'aide technique et à l'accompagnement fournis par les SADC ont souvent fait une différence dans la réussite de leur projet. Elles estiment toutefois avoir encore beaucoup de choses à apprendre en matière de gestion d'entreprise. 
Il est par ailleurs intéressant de constater que l'entrepreneuriat semble être un grand incitatif pour demeurer en région. En effet, pour la très grande majorité des femmes interviewées, le démarrage d'une entreprise les incitera à demeurer dans leur région. Plus encore, 15 femmes (38,5\%) auraient vraisemblablement quitté leur région si leur projet d'entreprise n'avait pas vu le jour.

Les intervenantes des SADC sont également très affirmatives à propos des retombées du projet pilote: sans le Fonds AFER Canada, plusieurs des entreprises créées n'auraient probablement pas pu être mises sur pied. Elles sont aussi d'avis qu'un très grand nombre de projets réussiront à passer la période difficile des deux premières années.

\section{Leçons à tirer}

L'accès au financement est un élément important de la problématique de l'entrepreneuriat féminin. Le Fonds AFER Canada a su cibler certains besoins des femmes entrepreneures et y apporter une réponse adéquate. Dans certains cas, le Fonds a fourni le coup de pouce qui manquait, l'aide supplémentaire qui a permis de motiver les entrepreneures, de les soutenir, de les conseiller et de les encourager à persévérer dans leur cheminement. Dans d'autres cas, le Fonds aura été l'élément déclencheur permettant de réaliser un projet qu'on n'aurait pas réalisé autrement.

Il semble par contre que les femmes aient besoin d'être encouragées pour démarrer leur projet d'entreprise. Dans les mois qui ont suivi la fin du projet pilote, le nombre de demandes d'aide aux SADC par des femmes a fortement décru. Il s'agit d'un premier indice suggérant que le développement de l'entrepreneuriat féminin en milieu rural doit s'appuyer sur un recrutement actif de candidates.

L'évaluation a révélé que la satisfaction du large éventail de besoins des femmes entrepreneures passe par l'échange et la complémentarité des organismes locaux dédiés au développement d'entreprises. Comme on a pu l'observer, plusieurs bénéficiaires du Fonds AFER Canada ont pu obtenir une aide complémentaire d'autres organismes, et ce, tant pour le développement de leur projet que pour le démarrage de leur entreprise. De l'aveu des personnes interrogées, cette synergie entre organismes locaux a souvent permis de trouver à proximité les services spécialisés dont avaient besoin les bénéficiaires.

L'exercice d'évaluation aura aussi permis de reconnaître l'apport inestimable de l'aide technique et de l'accompagnement pour celles qui veulent se faire entrepreneures. L'approche personnalisée et la proximité entre la bénéficiaire et sa conseillère semblent favoriser la confiance, la sécurité et l'engagement chez les femmes qui désirent démarrer une entreprise.

\section{Le Fonds AFER Canada a su cibler certains besoins des femmes entrepreneures et $y$ apporter une réponse adéquate}

\section{Conclusion}

Rappelons que si le projet pilote est maintenant terminé, la tâche des SADC participantes reste entière. Ces dernières devront être vigilantes et assurer un suivi auprès des bénéficiaires du Fonds AFER Canada. Si celui-ci a certes contribué à la création de plusieurs entreprises, encore faut-il qu'elles survivent. Or, le défi est encore plus grand puisque "l'échec d'une nouvelle entreprise est un destin plus probable que sa survie $»{ }^{9}$. Les nouvelles entreprises sont en effet les plus susceptibles de connaître des difficultés et, éventuellement, de cesser leurs activités. En ce sens, l'intervention des SADC en matière de suivi pourrait être déterminante dans le succès à long terme des entreprises créées dans le cadre du Fonds AFER Canada.

Bref, à la lumière de cette évaluation, le Fonds AFER Canada nous apparaît comme une intervention pouvant contribuer à consolider et à diversifier l'économie des régions rurales en misant sur la création d'entreprises par des femmes.

\section{Notes et références}

1. Groupe-conseil sur l'entrepreneuriat féminin (2000). Les défis des entrepreneures, Québec, Ministère de l'Industrie et du Commerce. 
2. Les auteurs tiennent à remercier le Réseau des Sociétés d'aide au développement des collectivités du Québec qui a autorisé l'utilisation des données de l'évaluation à des fins de publication

3. Administrée par la firme de sondage SOM, l'enquête a été effectuée en deux temps (mai 2005 et décembre 2005).

4. Les études de cas sont basées sur six entrevues semistructurées avec les représentantes des SADC concernées ainsi que des bénéficiaires du Fonds AFER Canada (entrevues réalisées en mars 2006).

5. Bien qu'un cadre de gestion précisait les grandes règles de fonctionnement du Fonds AFER, les SADC participantes avaient la possibilité d'adapter sa mise en œuvre à la réalité spécifique de leur région.

6. Selon le cadre de gestion du Fonds AFER Canada, les « niches rurales » renvoient à une stratégie de différenciation fondée territorialement sur la mise en valeur 1) d'un attribut original lié au territoire de référence, à une ressource, une caractéristique naturelle, un fait culturel ou historique ou 2) de caractéristiques particulières liées aux produits et services (matières premières de haute qualité ou uniques) et aux procédés utilisés (méthodes traditionnelles, procédés écologiques, etc.).

7. Ce nombre correspond aux entreprises dont le processus de démarrage n'était pas terminé au moment l'évaluation

8. Le nombre d'entreprises contenu dans ce tableau est différent du précédent puisqu'une bénéficiaire dont l'entreprise n'était pas encore démarrée pouvait, si elle possédait l'information, estimer le chiffre d'affaires prévu pour la première année.

9. Thornhill, Stewart et Raphael Amit (2003). Comprendre l'échec: mortalité organisationnelle et approche fondée sur les ressources, Ottawa, Statistiques Canada, p. v. 